\title{
THE NATURE OF HYPERVELOCITY STARS AND THE TIME BETWEEN THEIR FORMATION AND EJECTION
}

\author{
Warren R. Brown ${ }^{1}$, Judith G. Cohen ${ }^{2}$, Margaret J. Geller ${ }^{1}$, and Scott J. Kenyon ${ }^{1}$ \\ ${ }^{1}$ Smithsonian Astrophysical Observatory, 60 Garden Street, Cambridge, MA 02138, USA; \\ wbrown@cfa.harvard.edu,mgeller@cfa.harvard.edu, skenyon@cfa.harvard.edu \\ 2 Palomar Observatory, Mail Stop 249-17, California Institute of Technology, Pasadena, CA 91125, USA; jlc@ astro.caltech.edu \\ Received 2012 March 27; accepted 2012 June 7; published 2012 June 27
}

\begin{abstract}
We obtain Keck HIRES spectroscopy of HVS5, one of the fastest unbound stars in the Milky Way halo. We show that HVS5 is a $3.62 \pm 0.11 M_{\odot}$ main-sequence B star at a distance of $50 \pm 5 \mathrm{kpc}$. The difference between its age and its flight time from the Galactic center is $105 \pm 18$ (stat) \pm 30 (sys) Myr; flight times from locations elsewhere in the Galactic disk are similar. This $10^{8} \mathrm{yr}$ "arrival time" between formation and ejection is difficult to reconcile with any ejection scenario involving massive stars that live for only $10^{7} \mathrm{yr}$. For comparison, we derive arrival times of $10^{7} \mathrm{yr}$ for two unbound runaway B stars, consistent with their disk origin where ejection results from a supernova in a binary system or dynamical interactions between massive stars in a dense star cluster. For HVS5, ejection during the first $10^{7} \mathrm{yr}$ of its lifetime is ruled out at the $3 \sigma$ level. Together with the $10^{8} \mathrm{yr}$ arrival times inferred for three other well-studied hypervelocity stars (HVSs), these results are consistent with a Galactic center origin for the HVSs. If the HVSs were indeed ejected by the central black hole, then the Galactic center was forming stars $\simeq 200 \mathrm{Myr}$ ago, and the progenitors of the HVSs took $\simeq 100 \mathrm{Myr}$ to enter the black hole's loss cone.
\end{abstract}

Key words: Galaxy: center - Galaxy: halo - Galaxy: kinematics and dynamics - stars: early-type - stars: individual (SDSS J091759.47+672238.35)

Online-only material: color figures

\section{INTRODUCTION}

Hills (1988) first predicted unbound "hypervelocity" stars (HVSs) as the inevitable consequence of three-body interactions close to the tidal radius of a massive black hole. There is overwhelming evidence for a $4 \times 10^{6} M_{\odot}$ central black hole in the Milky Way (Ghez et al. 2008; Gillessen et al. 2009). Theorists expect that the black hole ejects $\sim 10^{-4} \mathrm{HVSs}_{\mathrm{yr}}{ }^{-1}$ (e.g., Perets et al. 2007), which means there are thousands of HVSs in the outer halo. Brown et al. (2005) discovered the first HVS, a luminous B-type star traveling twice the Galactic escape velocity at a distance of $\simeq 100 \mathrm{kpc}$, and Brown et al. (2012) have subsequently discovered 15 more unbound B-type stars in their targeted HVS survey. Establishing the evolutionary state of the HVSs is important for establishing their ages, distances, and flight times. We define the difference between an HVS's age and its flight time as the "arrival time" $\left(t_{\text {arr }}\right)$, the time between its formation and ejection. In this Letter, we derive $t_{\text {arr }}$ for both HVSs and unbound runaway stars.

The arrival time provides a useful discriminant between proposed ejection mechanisms. If HVSs are ejected in threebody interactions with the Milky Way's central black hole (Hills 1988), then the arrival times reflect the timescale for HVSs to achieve orbits that interact with the central black hole. For HVSs formed in the central region of the Galaxy, we expect $t_{\text {arr }}=0.1-1$ Gyr (Merritt \& Poon 2004; Wang \& Merritt 2004). On the other hand, in both mechanisms for ejecting runaway stars from the Galactic disk-a supernova in a binary system or a dynamical interaction among massive stars in a dense star cluster-a maximum $t_{\text {arr }} \approx 10 \mathrm{Myr}$ is set by the main-sequence lifetime of $\gtrsim 10 M_{\odot}$ stars. Thus, measuring $t_{\text {arr }}$ for an ensemble of HVSs should distinguish between a Galactic center and a Galactic disk origin.

The evolutionary state of most known HVSs (Brown et al. 2012) is ambiguous because their effective temperatures and surface gravities are consistent with both old, evolved stars (blue horizontal-branch stars) and short-lived main-sequence stars. Thus, we must turn to other measures to establish their nature. Metallicity is one possibility; we expect that recently formed stars should have solar or supersolar metallicities. Metallicity is inconclusive, however, given the observed metallicity distribution function of stars in the Milky Way.

Projected stellar rotation $v \sin i$ is a better discriminant between evolved stars and main-sequence stars. Blue horizontalbranch stars have evolved through the giant branch phase and have median $v \sin i=9 \mathrm{~km} \mathrm{~s}^{-1}$; the most extreme blue horizontal-branch star rotates at $40 \mathrm{~km} \mathrm{~s}^{-1}$ (Behr 2003). Late B-type main-sequence stars, on the other hand, have median $v \sin i=150 \mathrm{~km} \mathrm{~s}^{-1}$; the most extreme objects rotate at $\geqslant 350 \mathrm{~km} \mathrm{~s}^{-1}$ (Abt et al. 2002; Huang \& Gies 2006). Löckmann \& Baumgardt (2008) argue that HVSs may be spun up by a binary black hole ejection, but there is presently no evidence for a binary black hole in the Galactic center. Close binaries that produce HVSs and runaways in the Milky Way may exhibit slower stellar rotation because of tidal synchronization; Hansen (2007) predicts that late B-type HVSs ejected by the Hills mechanism should have $v \sin i=70-90 \mathrm{~km} \mathrm{~s}^{-1}$. In any case, fast rotation is the signature of a main-sequence star.

Of the B-type HVSs discovered to date, only HVS3, HVS7, and HVS8 have been studied with high-resolution spectroscopy. In all cases they are main-sequence B stars with $55 \mathrm{~km} \mathrm{~s}^{-1}<$ $v \sin i<260 \mathrm{~km} \mathrm{~s}^{-1}$ (Edelmann et al. 2005; Przybilla et al. 2008b, 2008c; Bonanos et al. 2008; López-Morales \& Bonanos 2008). Moderate-dispersion spectroscopy of HVS1 suggests it has $v \sin i=190 \mathrm{~km} \mathrm{~s}^{-1}$ (Heber et al. 2008b), another shortlived B star.

Here, we describe high-resolution spectroscopy of HVS5, a $g=17.9$ mag star located at declination $+67^{\circ}$ accessible only with Keck HIRES. HVS5 is a rapidly rotating $3.6 M_{\odot}$ mainsequence B star. The difference between its age and its flight 
time from the Milky Way is $105 \pm 18$ (stat) \pm 30 (sys) Myr, inconsistent with ejection models involving massive stars.

In Section 2, we describe the observations and stellar atmosphere analysis. In Section 3, we discuss the arrival times for the HVSs and unbound disk runaways. We conclude in Section 4.

\section{DATA}

\subsection{Observations}

We observed HVS5 using the HIRES spectrograph (Vogt et al. 1994) at the $10 \mathrm{~m}$ Keck I telescope, obtaining four 1800 s exposures the night of 2012 January 29, and five more the following night. Both nights were clear with $0.7-0.8^{\prime \prime}$ seeing. The red HIRES collimator was used in an instrument configuration that gave spectral coverage from 3920 to $8350 \AA$. A $1.1^{\prime \prime}$ slit gives a spectral resolution of $R=34,000$, with 6.7 pixels per spectral resolution element. There are small gaps between the three CCDs that form the detector mosaic, sometimes resulting in the loss of all or part of a single echelle order.

We also observed eight B stars selected from Abt et al. (2002) that span a wide range in luminosity class (I, III, and V) and a large range in projected rotational velocity $\left(5 \mathrm{~km} \mathrm{~s}^{-1}<\right.$ $v \sin i<285 \mathrm{~km} \mathrm{~s}^{-1}$ ). The stars are HR1328, HR1333, HR1399, HR1419, HR1420, HR1462, HR1573, HR1595, and HR1640. We used high signal-to-noise (S/N) spectra of these eight B stars to provide a comparison for HVS5 and to validate our analysis below.

We used the pipeline package MAKEE ${ }^{3}$ to remove the instrumental signature, extract a one-dimensional spectrum for each echelle order, and calibrate the wavelength scale from Th-A arc spectra taken at the beginning and end of each night. Each exposure of HVS5 was individually processed through MAKEE, and the results summed. With a total integration of $4.5 \mathrm{hr}$, we achieved an S/N of 70 per spectral resolution element at $4500 \AA$.

\subsection{Spectral Analysis}

Visual inspection reveals that HVS5 is a fast rotator. The FWHM of the MgII $\lambda 4481$ line compared with the Abt et al. (2002) B stars suggests a projected rotation velocity of $\simeq 130 \mathrm{~km} \mathrm{~s}^{-1}$. We turn to the most recent ATLAS9 model atmosphere grids (Castelli \& Kurucz 2003; Castelli et al. 1997) to perform a more quantitative analysis. We use the program SPECTRUM (Gray \& Corbally 1994) to calculate synthetic spectra under the assumption of plane-parallel atmosphere and local thermodynamic equilibrium. We adopt macro- and microturbulunce velocities of 0 and $2 \mathrm{~km} \mathrm{~s}^{-1}$, respectively. All synthetic spectra are smoothed to a resolution of $R=34,000$ and re-sampled with spline interpolation to match the sampling of the observed spectra.

Our approach is to analyze the spectral lines on an orderby-order basis. We normalize the continuum, calculate the $\chi^{2}$ of each synthetic model against the data, and then fit the resulting distribution of $\chi^{2}$ to derive the best-fitting parameters. Uncertainty estimates are provided by a standard $\Delta \chi^{2}$ offset to the minimum $\chi^{2}$ (Press et al. 1992). Our final values are the weighted means and error-on-the-means of the parameters measured from lines in different orders.

\footnotetext{
3 MAKEE was developed by T. A. Barlow specifically for reduction of Keck HIRES data. It is freely available from the Keck HIRES home page www2.keck.hawaii.edu/inst/hires.
}

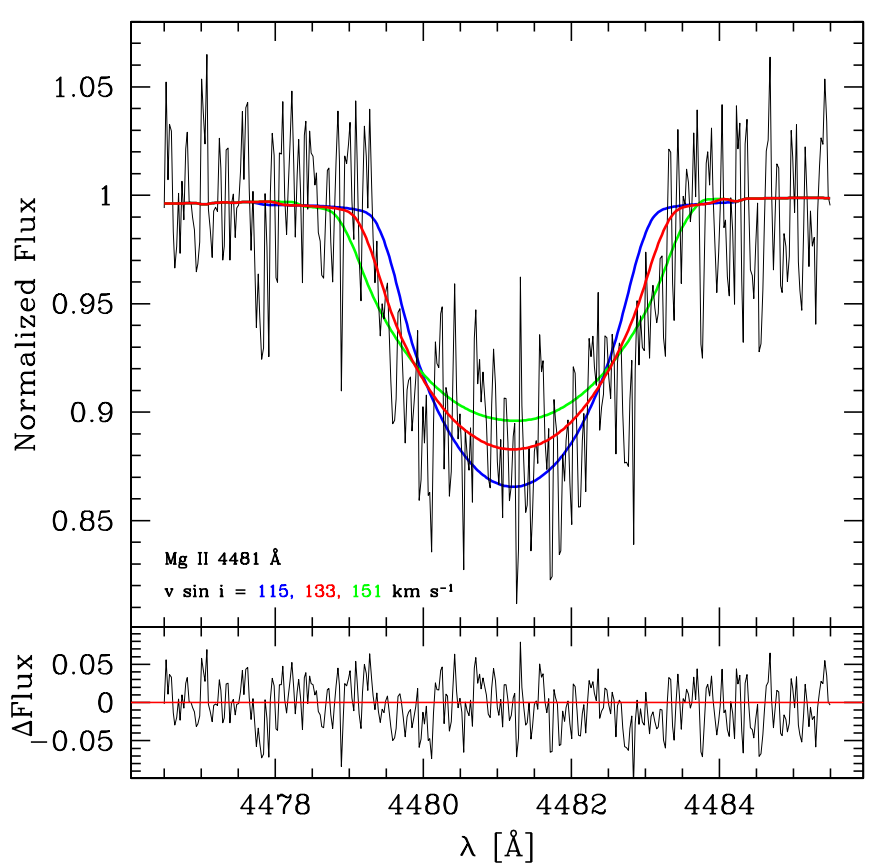

Figure 1. Observed $\mathrm{Mg}$ II $\lambda 4481$ line (upper panel) and its residuals (lower panel) compared to the best-fit $v \sin i=133 \pm 7 \mathrm{~km} \mathrm{~s}^{-1}$ model (red line). For reference, the green and blue lines are the $\pm 3 \sigma$ models.

(A color version of this figure is available in the online journal.)

We begin by using all of the spectral lines to solve for the heliocentric radial velocity. The best-fit $+552 \pm 3 \mathrm{~km} \mathrm{~s}^{-1}$ velocity is in perfect agreement with the $+553 \pm 9 \mathrm{~km} \mathrm{~s}^{-1}$ velocity measured from medium-resolution spectroscopy at the MMT (Brown et al. 2012). The constancy in velocity is consistent with HVS5 being a single star, as one expects for the product of a binary disruption. The radial velocity corresponds to a minimum velocity of $+663 \mathrm{~km} \mathrm{~s}^{-1}$ in the Galactic rest frame (see Brown et al. 2012).

Next, we measure projected rotation using $\mathrm{Mg}$ II $\lambda 4481$, the strongest metal line in the spectrum (see Figure 1). The best-fit $v \sin i$ is $133 \pm 7 \mathrm{~km} \mathrm{~s}^{-1}$. For comparison, Figure 1 plots the $\pm 3 \sigma$ values as well as the residuals to the best-fit $v \sin i$. The observed $v \sin i$ is consistent with the median $v \sin i$ of comparable B-type main-sequence stars (Abt et al. 2002; Huang \& Gies 2006).

Given the observed $v \sin i$, we measure effective temperature and surface gravity by fitting the widths and depths of the $T_{\text {eff- }}$ and $\log g$-sensitive hydrogen Balmer lines. We note that the model hydrogen lines are computed using the D. Peterson routine adopted by SYNTHE (Kurucz 1993), which includes Stark and resonance broadening and fine structure in the cores. The best-fit values are $T_{\text {eff }}=12,000 \pm 350 \mathrm{~K}$ and $\log g=$ $3.89 \pm 0.13$; Figure 2 compares the best-fit model with the data.

Finally, we attempt to constrain the metallicity. Because of the large $v \sin i$, Fe lines are faint continuum fluctuations and are thus too weak to provide significant constraint. Si II lines at $\lambda 4128, \lambda 4131$, and $\lambda 5056$ are stronger and yield a best-fit $\mathrm{Si}$ abundance of $[\mathrm{M} / \mathrm{H}]=-0.4 \pm 0.5$. The Mg II $\lambda 4481$ line (see Figure 1), on the other hand, yields a best-fit $\mathrm{Mg}$ abundance of $[\mathrm{M} / \mathrm{H}]=+0.3 \pm 0.5$. Given the large uncertainties, we conclude that HVS5 is consistent with solar abundance.

Figure 3 compares the measured $T_{\text {eff }}$ and $\log g$ with the latest Girardi et al. $(2002,2004)$ solar metallicity main-sequence tracks. The ellipse in Figure 3 is the $68.3 \%(1 \sigma)$ confidence region. Interpolating the tracks indicates that HVS5 is a 


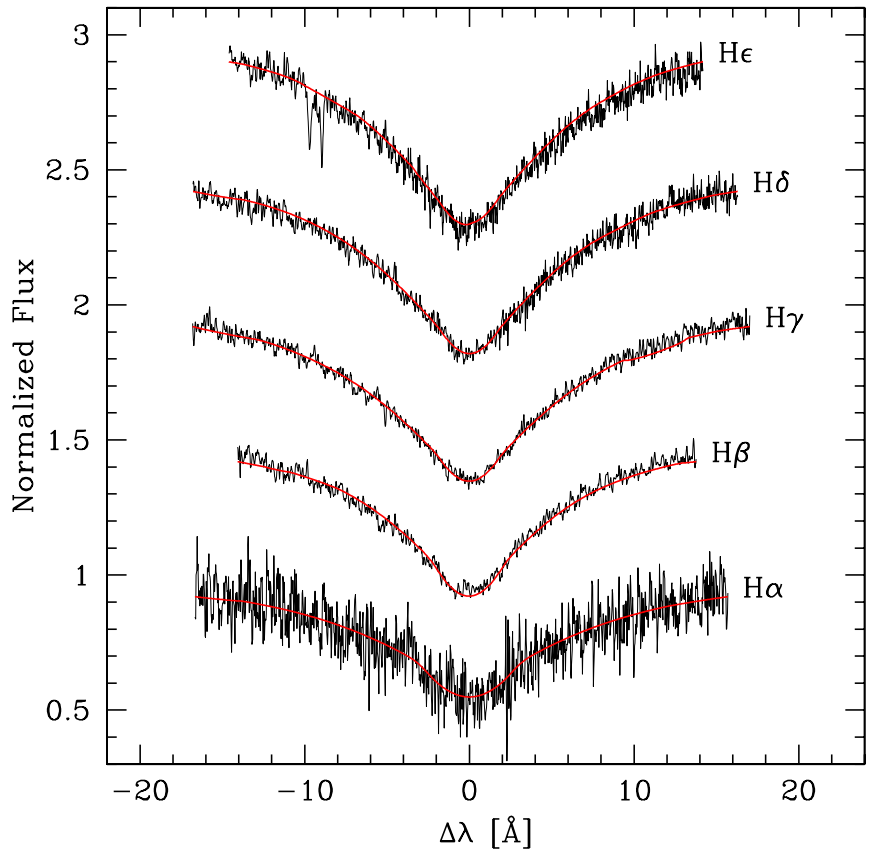

Figure 2. Observed hydrogen Balmer lines compared to the best-fit model (red line). The temperature- and surface-gravity-sensitive lines give best-fit values of $T_{\text {eff }}=12,000 \pm 350 \mathrm{~K}$ and $\log g=3.89 \pm 0.13$.

(A color version of this figure is available in the online journal.)

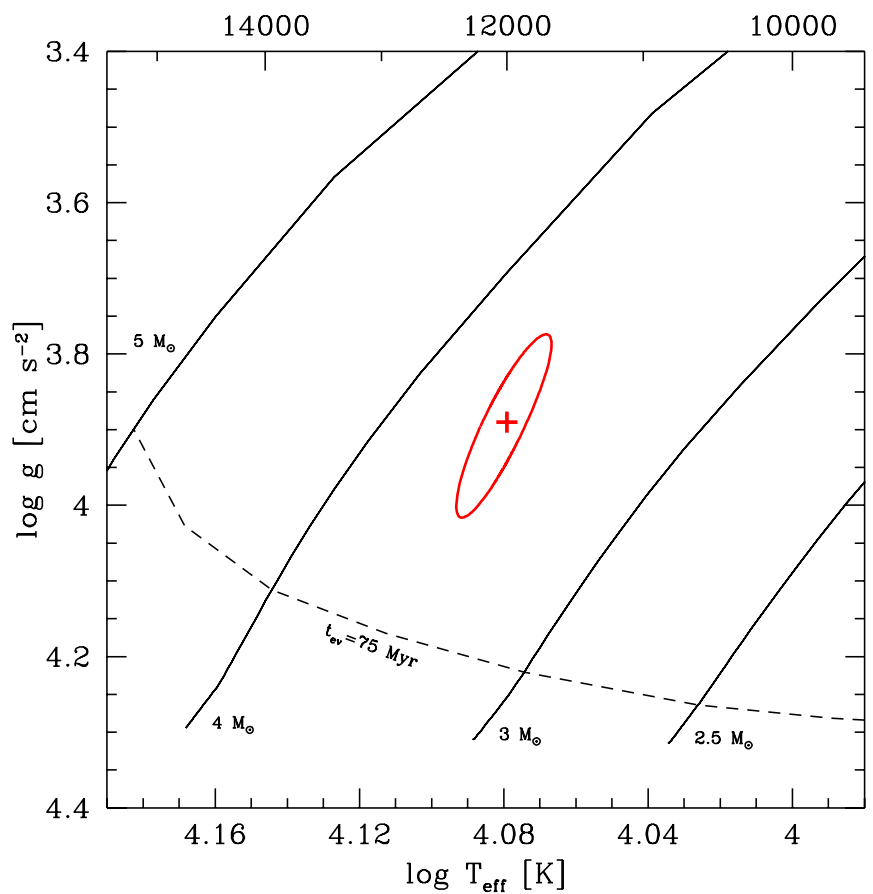

Figure 3. Measured $T_{\text {eff }}, \log g$, and the $1 \sigma$ error ellipse for HVS5 (in red) compared to Girardi et al. $(2002,2004)$ solar metallicity main-sequence tracks for 2.5-5 $M_{\odot}$ stars (solid black lines); the $t_{e v}=75 \mathrm{Myr}$ isochrone is plotted for reference. HVS5 is a $3.62 \pm 0.11 M_{\odot}$ star.

(A color version of this figure is available in the online journal.)

$3.62 \pm 0.11 M_{\odot}$ star. As an illustration of the systematic uncertainty, we derive $3.58 M_{\odot}$ from Ekström et al. (2012) tracks with rotation, and $3.72 M_{\odot}$ from Girardi et al. $(2002,2004)+0.2$ dex supersolar tracks. These values are consistent within our $1 \sigma$ uncertainty, thus the inferred mass is relatively insensitive to rotation and metallicity.

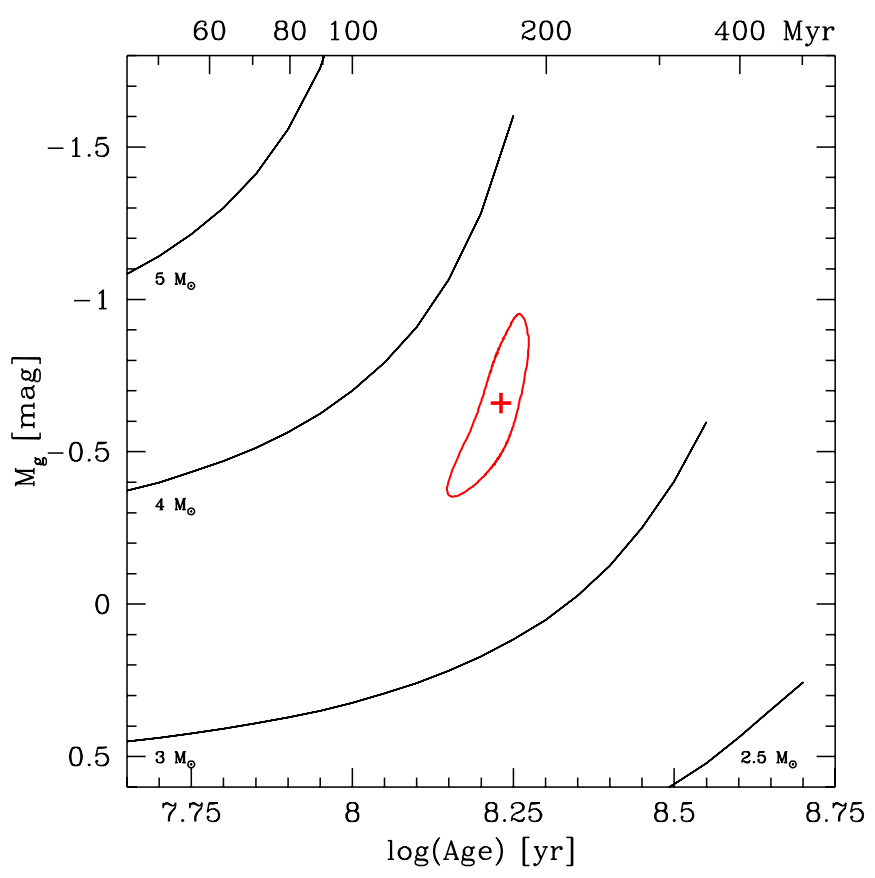

Figure 4. Same tracks as Figure 3 but now plotting age vs. absolute magnitude. HVS5 has a formal age of $170 \pm 17$ (stat) \pm 30 (sys) Myr.

(A color version of this figure is available in the online journal.)

Table 1 summarizes our stellar parameters for HVS5. We also list the parameters for HVS7, HVS8, and HVS1 measured by Przybilla et al. (2008c), López-Morales \& Bonanos (2008), and Heber et al. (2008b), respectively. HVS3, a probable blue straggler (Brown et al. 2010), is not directly comparable and is not included in our discussion.

\section{DISCUSSION}

\subsection{Hypervelocity Star Flight Times and Ages}

We use the Girardi et al. $(2002,2004)$ tracks to derive age and luminosity from the measured $T_{\text {eff }}$ and $\log g$. We note that the $T_{\text {eff }}-\log g$ error ellipse becomes a banana shape in the age $-M_{g}$ plot (Figure 4) because of the time evolution of these parameters. Interpolating the tracks indicates that HVS5 has an age of $170 \pm 17 \mathrm{Myr}$ and an absolute magnitude of $M_{g}=-0.65 \pm 0.19$.

Knowing the luminosity of HVS5, we can calculate its distance and flight time. HVS5 has an apparent de-reddened magnitude of $g=17.557 \pm 0.021$ and thus a heliocentric distance of $44 \pm 4 \mathrm{kpc}$. Assuming the Sun is located $8 \mathrm{kpc}$ from the Galactic center, HVS5 has a Galactocentric distance of $r_{\mathrm{GC}}=50 \pm 5 \mathrm{kpc}$. We then take the Galactic potential model of Kenyon et al. (2008) and calculate all possible trajectories that HVS5 could have given its present distance and radial velocity. The trajectory that passes through the Galactic center has a flight time of $t_{\mathrm{GC}}=65 \pm 7 \mathrm{Myr}$. The error comes from propagating the distance and radial velocity errors through the trajectory calculation.

Our Galactic center flight time estimate is appropriate for a wide range of Milky Way starting locations because HVS5 is located at high Galactic latitude and at large distance. Moving the assumed starting location of HVS5 from $r_{\mathrm{GC}}=0 \mathrm{kpc}$ to $r_{\mathrm{GC}}=10 \mathrm{kpc}$ changes the flight times by $\pm 8.5 \mathrm{Myr}$, which is similar to the estimated uncertainty in flight time.

For self-consistency, we also derive the ages and flight times of HVS7, HVS8, and HVS1 using the same tracks and 
Table 1

Hypervelocity Star Properties

\begin{tabular}{|c|c|c|c|c|}
\hline Property & HVS5 & HVS7 & HVS8 & HVS1 \\
\hline$T_{\text {eff }}(\mathrm{K})$ & $12000 \pm 350$ & $12000 \pm 500$ & $11000 \pm 1000$ & $11000 \pm 500$ \\
\hline $\log g(\mathrm{cgs})$ & $3.89 \pm 0.13$ & $3.8 \pm 0.1$ & $3.75 \pm 0.25$ & $3.74 \pm 0.20$ \\
\hline$v \sin i\left(\mathrm{~km} \mathrm{~s}^{-1}\right)$ & $133 \pm 7$ & $55 \pm 2$ & $260 \pm 70$ & $190 \pm 40$ \\
\hline Mass $\left(M_{\odot}\right)$ & $3.62 \pm 0.11$ & $3.79 \pm 0.09$ & $3.49 \pm 0.22$ & $3.50 \pm 0.18$ \\
\hline Age (Myr) & $170 \pm 17$ & $170 \pm 15$ & $220 \pm 25$ & $220 \pm 20$ \\
\hline$M_{g}(\mathrm{mag})$ & $-0.65 \pm 0.19$ & $-0.94 \pm 0.14$ & $-0.77 \pm 0.35$ & $-0.80 \pm 0.29$ \\
\hline$g_{0}(\mathrm{mag})$ & $17.557 \pm 0.021$ & $17.637 \pm 0.019$ & $17.939 \pm 0.016$ & $19.688 \pm 0.023$ \\
\hline$r_{\mathrm{GC}}(\mathrm{kpc})$ & $50 \pm 5$ & $53 \pm 4$ & $60 \pm 10$ & $130 \pm 18$ \\
\hline$t_{\mathrm{GC}}(\mathrm{Myr})$ & $65 \pm 7$ & $105 \pm 10$ & $120 \pm 20$ & $175 \pm 25$ \\
\hline$t_{\mathrm{arr}}=\mathrm{Age}-t_{\mathrm{GC}}(\mathrm{Myr})$ & $105 \pm 18$ & $65 \pm 18$ & $100 \pm 32$ & $45 \pm 32$ \\
\hline
\end{tabular}

methodology. Table 1 summarizes the derived values. The HVSs have ages of 170-220 Myr and flight times of 45-175 Myr.

\subsection{Links to Unbound Ejection Processes}

There are many ways to eject stars from their place of origin, but few processes can accelerate stars to unbound velocities. Because most B stars are binaries (e.g., Chini et al. 2012), disk "runaway" B stars are explained by binary disruption mechanisms. In the case of a supernova in a binary system, the timescale of the process is the lifetime of the $\gtrsim 10 M_{\odot}$ star that explodes, $10^{6}-10^{7} \mathrm{yr}$. In the case of dynamical three- and four-body encounters, e.g., in young star clusters, massive stars are necessary to attain the unbound velocities of HVSs and thus the timescale of the dynamical process is also $\simeq 10^{7}$ yr. Except in rare circumstances (Gualandris \& Portegies Zwart 2007; Gvaramadze et al. 2009; Silva \& Napiwotzki 2011), no runaway mechanism is expected to yield unbound velocities for $3 M_{\odot}$ stars (Portegies Zwart 2000; Perets \& Subr 2012).

A more energetic and higher ejection rate process exists in the Galactic center: HVSs ejected by the central black hole (Hills 1988). The B stars that presently orbit Sgr A* on short-period, eccentric orbits are, in this scenario, the former companions of HVSs; their progenitors are believed to have formed farther out and then moved in toward the black hole through dynamical processes (e.g., Perets et al. 2007, 2009; Madigan et al. 2009, 2011).

In principle, there is no upper limit to the arrival time $t_{\text {arr }}$ for the central black hole ejection process. The black hole is always there, and ongoing star formation (e.g., Lu et al. 2009) provides a constant supply of new stars. To derive a typical $t_{\text {arr }}$, we consider the "loss cone," the set of orbits which have a distance of closest approach within the black hole's tidal radius. For an ensemble of stars formed close to the black hole, a few will have orbits that interact with the black hole on $\lesssim 1$ Myr timescales and so are quickly removed. As a result of dynamical interactions with other massive objects or the long-term evolution of chaotic orbits within a triaxial potential, the remaining stars will "fill" the loss cone with timescales of 100 Myr to 1 Gyr (Yu \& Tremaine 2003; Merritt \& Poon 2004; Wang \& Merritt 2004; Perets et al. 2007).

Timescale thus provides an important distinction between the central black hole and disk runaway ejection processes. The disk runaway scenarios must eject stars within the 1-10 Myr lifetimes of massive stars to attain unbound velocities. The central black hole can eject unbound stars at any time; however, we expect that stars formed in the Galactic center will have typical arrival times of 0.1-1 Gyr. For the HVSs studied here, an upper limit is provided by their finite lifetimes.

\subsection{Comparison with Observations}

From Table 1 , observed HVSs have $t_{\text {arr }} \approx 50-100$ Myr. The formal error in $t_{\text {arr }}$ is likely an underestimate of the true error, however. Perhaps the best estimate of systematic error comes from comparing the measured stellar parameters with different sets of stellar evolution tracks. For HVS5, the Ekström et al. (2012) tracks for rotating stars give a longer age of $200 \pm 23$ Myr, while the Girardi et al. $(2002,2004)+0.2$ dex supersolar tracks give a shorter age of $142 \pm 16$ Myr. Taking the \pm 30 Myr age spread as the systematic error rules out an ejection in the first $10 \mathrm{Myr}$ of HVS5's lifetime at the $3 \sigma$ level. This confidence level is corroborated by the directly measured parameters: the $\log g$ and $T_{\text {eff }}$ of a $75 \mathrm{Myr}$ old $3.62 M_{\odot}$ star differ by $2.1 \sigma$ and $3.3 \sigma$, respectively, with respect to HVS5's present values (see Figure 3 ). Thus, for HVS5 we rule out a possible $t_{\text {arr }} \lesssim 10 \mathrm{Myr}$ at the $3 \sigma$ level, an interesting and important constraint on its origin.

The hyperrunaways first discovered by Heber et al. (2008a) show a contrasting result. HD 271791 is an unbound $11 M_{\odot} \mathrm{B}$ star. The observed proper motion shows it was ejected in the direction of rotation from the outer disk (Heber et al. 2008a). The star has an age of $25 \pm 5 \mathrm{Myr}$ and a flight time from the disk of $25 \pm 6 \mathrm{Myr}$. Formally, the star has $t_{\text {arr }}=0 \pm 8 \mathrm{Myr}$. The marginally unbound $5 M_{\odot}$ B star HIP 60350 is similar (Irrgang et al. 2010). It has an age of $45_{-30}^{+15} \mathrm{Myr}$ and a flight time from the disk of $14 \pm 3$ Myr. Thus, $t_{\text {arr }}=31_{-30}^{+15}$ Myr. The short arrival times are consistent with both the supernova ejection scenario (Przybilla et al. 2008a) and the dynamical ejection scenario (Gvaramadze 2009). Contrasting the derived arrival times with arrival times for HVSs underscores the usefulness of $t_{\text {arr }}$ as a model discriminant.

Other objects are more ambiguous. Tillich et al. (2009) discovered the marginally unbound $2.5 M_{\odot}$ A star J0136+2425 with a derived age of $245 \mathrm{Myr}$ and a flight time of $12 \mathrm{Myr}$ if it comes from the disk. Accepting modern Milky Way halo mass estimates of $\simeq 1.7 \times 10^{12} M_{\odot}$ (e.g., Gnedin et al. 2010; Przybilla et al. 2010), it is bound to the Milky Way and may be explained as a halo star. The evolved sdB star J1211+1437 has a flight time that is also a small fraction of its progenitor's lifetime (which may be many Gyr; Tillich et al. 2011). Given the $\pm 140 \mathrm{~km} \mathrm{~s}^{-1}$ uncertainty in the space motion, this sdB star is also consistent with being bound and thus a normal halo star.

The HVSs are significantly unbound based on radial velocity alone. The four HVSs with known evolutionary state discussed here have $t_{\text {arr }}=50-100 \mathrm{Myr}$, times both larger than known hyperrunaways and larger than the maximum $t_{\text {arr }}$ expected in the mechanisms for producing hyperrunaways. However, their $t_{\text {arr }}$ are close to the arrival times expected for 
dynamical interactions with the black hole at the Galactic center.

\section{CONCLUSION}

We describe Keck HIRES spectroscopy of HVS5, one of the fastest known HVSs with a minimum Galactic rest-frame velocity of $+663 \pm 3 \mathrm{~km} \mathrm{~s}^{-1}$. The observations reveal that HVS5 has a projected rotation of $v \sin i=133 \pm 7 \mathrm{~km} \mathrm{~s}^{-1}$ and is thus a main-sequence B star. Comparing the measured $T_{\text {eff }}$ and $\log g$ with stellar evolution tracks indicates that HVS5 is a $3.62 \pm 0.11 M_{\odot}, 170 \pm 17 \mathrm{Myr}$ old star. Given its present distance and radial velocity, we calculate that HVS5's arrival time, the time between its formation and subsequent ejection, is $t_{\text {arr }}=105 \pm 18$ (stat) \pm 30 (sys) Myr.

This timescale provides an interesting new constraint on the origin of unbound runaways and HVSs. Runaway B stars near the disk have $t_{\text {arr }}=0-30 \mathrm{Myr}$, consistent with disk ejection scenarios involving a supernova in a binary system or a dynamical event among several massive stars. The set of B-type HVSs with known evolutionary states, on the other hand, have $t_{\text {arr }}=50-100$ Myr. This timescale is difficult to reconcile with any ejection mechanism requiring a massive star to attain unbound ejection. The central black hole ejection scenario, however, allows for any $t_{\text {arr }}$. Thus, the derived arrival times for HVSs support the black hole ejection model.

Future progress requires obtaining high-resolution observations of other HVSs to constrain their age and distance. The age distribution of HVSs has important implications for the epochs of star formation and the growth of the central black hole (Bromley et al. 2012). Combined with future proper motion measurements, we hope to directly constrain the full space velocity and place of origin of the HVSs.

This work was supported in part by the Smithsonian Institution. J. Cohen acknowledges partial support from NSF grant AST-0908139. This research makes use of NASA's Astrophysics Data System Bibliographic Services. We are grateful to the many people who have worked to make the Keck Telescopes and their instruments a reality, and who operate and maintain these observatories. The authors extend special thanks to those of Hawaiian ancestry on whose sacred mountain we are privileged to be guests. Without their generous hospitality, none of the observations presented herein would have been possible

\section{REFERENCES}

Abt, H. A., Levato, H., \& Grosso, M. 2002, ApJ, 573, 359

Behr, B. B. 2003, ApJS, 149, 67

Bonanos, A. Z., López-Morales, M., Hunter, I., \& Ryans, R. S. I. 2008, ApJ, 675, L77

Bromley, B. C., Kenyon, S. J., Geller, M. J., \& Brown, W. R. 2012, ApJ, 749, L42
Brown, W. R., Anderson, J., Gnedin, O. Y., et al. 2010, ApJ, 719, L23

Brown, W. R., Geller, M. J., \& Kenyon, S. J. 2012, ApJ, 751, 55

Brown, W. R., Geller, M. J., Kenyon, S. J., \& Kurtz, M. J. 2005, ApJ, 622, L33

Castelli, F., Gratton, R. G., \& Kurucz, R. L. 1997, A\&A, 318, 841

Castelli, F., \& Kurucz, R. L. 2003, in IAU Symp. 210, Modelling of Stellar Atmospheres, ed. N. Piskunov, W. W. Weiss, \& D. F. Gray (San Francisco, CA: ASP), poster A20

Chini, R., Hoffmeister, V. H., Nasseri, A., Stahl, O., \& Zinnecker, H. 2012, MNRAS, in press (arXiv:1205.5238)

Edelmann, H., Napiwotzki, R., Heber, U., Christlieb, N., \& Reimers, D. 2005, ApJ, 634, L181

Ekström, S., Georgy, C., Eggenberger, P., et al. 2012, A\&A, 537, A146

Ghez, A. M., Salim, S., Weinberg, N. N., et al. 2008, ApJ, 689, 1044

Gillessen, S., Eisenhauer, F., Trippe, S., et al. 2009, ApJ, 692, 1075

Girardi, L., Bertelli, G., Bressan, A., et al. 2002, A\&A, 391, 195

Girardi, L., Grebel, E. K., Odenkirchen, M., \& Chiosi, C. 2004, A\&A, 422, 205

Gnedin, O. Y., Brown, W. R., Geller, M. J., \& Kenyon, S. J. 2010, ApJ, 720, L108

Gray, R. O., \& Corbally, C. J. 1994, AJ, 107, 742

Gualandris, A., \& Portegies Zwart, S. 2007, MNRAS, 376, L29

Gvaramadze, V. V. 2009, MNRAS, 395, L85

Gvaramadze, V. V., Gualandris, A., \& Portegies Zwart, S. 2009, MNRAS, 396, 570

Hansen, B. M. S. 2007, ApJ, 671, L133

Heber, U., Edelmann, H., Napiwotzki, R., Altmann, M., \& Scholz, R.-D. 2008a, A\&A, 483, L21

Heber, U., Hirsch, H. A., Edelmann, H., et al. 2008b, in ASP Conf. Ser. 392, Hot Subdwarf Stars and Related Objects, ed. U. Heber, C. S. Jeffery, \& R Napiwotzki (San Francisco, CA: ASP), 167

Hills, J. G. 1988, Nature, 331, 687

Huang, W., \& Gies, D. R. 2006, ApJ, 648, 580

Irrgang, A., Przybilla, N., Heber, U., Fernanda Nieva, M., \& Schuh, S. 2010, ApJ, 711,138

Kenyon, S. J., Bromley, B. C., Geller, M. J., \& Brown, W. R. 2008, ApJ, 680, 312

Kurucz, R. L. 1993, SYNTHE Spectrum Synthesis Programs and Line Data (Kurucz CD-ROM; Cambridge, MA: Smithsonian Astrophysical Observatory)

Löckmann, U., \& Baumgardt, H. 2008, MNRAS, 384, 323

López-Morales, M., \& Bonanos, A. Z. 2008, ApJ, 685, L47

Lu, J. R., Ghez, A. M., Hornstein, S. D., et al. 2009, ApJ, 690, 1463

Madigan, A.-M., Hopman, C., \& Levin, Y. 2011, ApJ, 738, 99

Madigan, A.-M., Levin, Y., \& Hopman, C. 2009, ApJ, 697, L44

Merritt, D., \& Poon, M. Y. 2004, ApJ, 606, 788

Perets, H. B., Gualandris, A., Merritt, D., \& Alexander, T. 2009, ApJ, 702, 884

Perets, H. B., Hopman, C., \& Alexander, T. 2007, ApJ, 656, 709

Perets, H. B., \& Subr, L. 2012, ApJ, 751, 133

Portegies Zwart, S. F. 2000, ApJ, 544, 437

Press, W. H., Teukolsky, S. A., Vetterling, W. T., \& Flannery, B. P. 1992, Numerical recipes in C: The Art of Scientific Computing (2nd ed.; Cambridge: Cambridge Univ. Press)

Przybilla, N., Nieva, M. F., Heber, U., \& Butler, K. 2008a, ApJ, 684, L103

Przybilla, N., Nieva, M. F., Heber, U., et al. 2008b, A\&A, 480, L37

Przybilla, N., Nieva, M. F., Tillich, A., et al. 2008c, A\&A, 488, L51

Przybilla, N., Tillich, A., Heber, U., \& Scholz, R.-D. 2010, ApJ, 718, 37

Silva, M. D. V., \& Napiwotzki, R. 2011, MNRAS, 411, 2596

Tillich, A., Heber, U., Geier, S., et al. 2011, A\&A, 527, A137

Tillich, A., Przybilla, N., Scholz, R., \& Heber, U. 2009, A\&A, 507, L37

Vogt, S. S., Allen, S. L., Bigelow, B. C., et al. 1994, Proc. SPIE, 2198, 362

Wang, J., \& Merritt, D. 2004, ApJ, 600, 149

Yu, Q., \& Tremaine, S. 2003, ApJ, 599, 1129 